\title{
On the Stabilizability of Two-dimensional Linear Systems via Switched Output Feedback
}

Keith R. Santarelli

\author{
Alexandre Megretski \\ Laboratory for Information and Decision Systems \\ Massachusetts Institute of Technology \\ Cambridge, MA, 02139, USA \\ Email: \{krsanta, ameg, dahleh\}@ @it.edu
}

\author{
Munther A. Dahleh
}

\begin{abstract}
The problem of stabilizing a second order SISO LTI system of the form $\dot{x}=A x+B u, y=C x$ with feedback of the form $u(x)=v(x) C x$ is considered, where $v(x)$ is realvalued and has domain which is all of $\mathbf{R}^{2}$. It is shown that, when stabilization is possible, $v(x)$ can be chosen to take on no more than two values throughout the entire state space (i.e., $v(x) \in\left\{v_{1}, v_{2}\right\}$ for all $x$ and for some $v_{1}, v_{2}$ ), and an algorithm for finding a specific choice of $v(x)$ is presented. It is also shown that the classical root locus of the corresponding transfer function $C(s I-A)^{-1} B$ has a strong connection to this stabilization problem, and its utility is demonstrated through several design examples.
\end{abstract}

\section{INTRODUCTION}

Stabilization of continuous time systems via hybrid feedback (in which a controller which possesses both continuous and discrete dynamics is employed) is a problem that has received much attention in the recent literature. Artstein first raised this question via examples [1]. Litsyn et. al. show in [2] that the linear system

$$
\dot{x}=A x+B u, \quad y=C x
$$

with $(A, B)$ reachable and $(C, A)$ observable can be stabilized via a hybrid feedback controller which uses a countable number of discrete states (and no continuous states) and which only depends upon the output $y$ as opposed to the entire continuous state $x$. A natural question arises as to whether a hybrid feedback controller can be designed which uses a finite number of states instead. For the most part, the answer to this question is still open, though a partial answer has been given by $\mathrm{Hu}$ et. al. in [3] based upon the so-called conic switching laws of [4] and [5]. In [3], it is shown that, for a certain class of single-input, singleoutput (SISO) second order systems which are reachable and observable, there exists a feedback control law of the form $u(x)=v(x) C x$ where

$$
v(x)= \begin{cases}v_{1}, & \text { if } x_{1} x_{2} \geq 0 \\ v_{2}, & \text { if } x_{1} x_{2}<0\end{cases}
$$

with $x=\left[\begin{array}{ll}x_{1} & x_{2}\end{array}\right]^{\prime}$ such that the resulting closed-loop system

$$
\dot{x}=A x+v(x) B C x
$$

is globally exponentially stable. A control law of the form I.2 is desirable as it can be implemented as a switch between two static gains which multiplies the output $y=C x$. Note that, in general, the above strategy does not always work as the result of [2] sometimes requires a more complicated hybrid feedback structure to achieve stability, even when the system described by I.1 is reachable and observable.

Example I.1: Consider I.1 with

$$
A=\left[\begin{array}{rr}
2 & -1 \\
-1 & 2
\end{array}\right], B=\left[\begin{array}{l}
0 \\
1
\end{array}\right], C=\left[\begin{array}{ll}
0 & 1
\end{array}\right] .
$$

The system is reachable and observable, but I.3 is not stable for any real-valued choice of $v(x) \equiv v\left(x_{1}, x_{2}\right)$ whose domain is all of $\mathbf{R}^{2}$, not just $v\left(x_{1}, x_{2}\right)$ of the form I.2. ${ }^{1}$ To see this, first note that the region $x_{1}<0, x_{2}>0$ is invariant under the flow of I.3 for any choice of $v\left(x_{1}, x_{2}\right)$. Indeed, when $x_{1}=0, \dot{x}_{1}=-x_{2}<0$, and when $x_{2}=0$, $\dot{x}_{2}=-x_{1}>0$ for all choices of $v(x)$. Moreover, when $x_{1}(0)<0$ and $x_{2}(0)>0, \dot{x}_{1}=2 x_{1}-x_{2}<0$, which means that $x_{1}(t)$ is strictly decreasing, and, hence, does not decay to zero regardless of the choice of $v\left(x_{1}, x_{2}\right)$.

The goal of this paper is to answer the following questions: under what conditions on $A \in \mathbf{R}^{2 \times 2}, B \in \mathbf{R}^{2 \times 1}$ and $C \in \mathbf{R}^{1 \times 2}$ can the closed-loop system I.3 be made asymptotically stable for some choice of $v\left(x_{1}, x_{2}\right)$ ? And, moreover, when stability is achievable, how may one design $v\left(x_{1}, x_{2}\right)$ explicitly? As it turns out, the answer to the first question has a strong connection to the classical control notion of root locus. Essentially, if one considers control laws of the form $v\left(x_{1}, x_{2}\right)=k$ for some constant $k$ where $k$ varies continuously over $\mathbf{R}$, then the system I.3 is stabilizable in only one of two situations:

- There exists a value of $k$ such that the matrix $A+k B C$ is Hurwitz and, hence, I.3 is exponentially stabilizable via static output feedback.

- There is no value of $k$ for which $A+k B C$ is Hurwitz, but there does exist a value of $k$ for which the eigenvalues of $A+k B C$ are complex. In this case, $v\left(x_{1}, x_{2}\right)$

\footnotetext{
${ }^{1}$ We will implicitly make this assumption on the domain of $v(x)$ throughout the paper. Note that this eliminates choices of $v(x)$ which blow up for some value(s) of $x$, such as $v(x)$ which attempt to divide by the output $y=C x$.
} 
can be chosen to take on only two values $v_{1}$ and $v_{2}$ throughout the entire state-space, i.e., $v\left(x_{1}, x_{2}\right) \in$ $\left\{v_{1}, v_{2}\right\}$, where $v_{1}$ and $v_{2}$ are appropriately selected real constants, and global exponential stability can be achieved.

A third situation can exist in which there exists no value of $k$ for which $A+k B C$ is Hurwitz and the eigenvalues of $A+k B C$ are real for all $k$. It is precisely these situations for which no choice of $v\left(x_{1}, x_{2}\right)$ will yield asymptotic stability.

Note that, unlike [2], the switching strategies employed here and in [3] in general require full knowledge of the state $x$ of I. 1 rather than just knowing the output $y=C x$. Nevertheless, finite state automata can be designed which practically implement the switching strategies presented here and in [3], though we will not formally show this here. The main goal of this work is to provide theoretical limitations as to which SISO second order systems of the form I.1 do not admit themselves to a stabilizing hybrid controller which attempts to implement the switching law of I.2.

The structure of the paper is as follows. First, we examine two particular case studies in which the form of the $B$ and $C$ vectors have special structure and analyze the conditions on the matrix $A$ which will guarantee stability. Also, we will derive explicit forms for $v\left(x_{1}, x_{2}\right)$ which can be used to achieve stability when it is possible to do so. Next, we will show that, through appropriate coordinate transformations, all nontrivial ${ }^{2}$ problems can be transformed into either one of these two case studies and then will use this to establish the main result. Finally, we explore a general method of designing such controllers (when they exist) and provide several examples to illustrate the methodology.

\section{CASE Studies}

In this section, we explore two specific case studies in which the $A, B$ and $C$ matrices of I.1 have particular structures. Using appropriate coordinate transformations, we will then relate the results of this section to derive the main result for general $A, B$, and $C$.

\section{A. Case 1}

We first assume a system of the following structure:

$$
A=\left[\begin{array}{ll}
a & b \\
c & 0
\end{array}\right], B=\left[\begin{array}{l}
0 \\
1
\end{array}\right], C=\left[\begin{array}{ll}
0 & 1
\end{array}\right],
$$

where $a, c \in \mathbf{R}$, and $b \geq 0$. Here, I.3 takes the form

$$
\left[\begin{array}{l}
\dot{x}_{1} \\
\dot{x}_{2}
\end{array}\right]=\left[\begin{array}{cc}
a & b \\
c & v\left(x_{1}, x_{2}\right)
\end{array}\right]\left[\begin{array}{l}
x_{1} \\
x_{2}
\end{array}\right]
$$

We summarize the possibilities for stabilizability as a function of the parameters $a, b$, and $c$ in the proposition below:

Proposition II.1: For the system II.5:

\footnotetext{
${ }^{2}$ By "nontrivial", we refer to problems in which neither $B$ nor $C$ is identically 0 .
}

1) If $b c=0$, then II.5 is exponentially stabilizable via static output feedback if $a<0$ and is not stabilizable for any choice of $v\left(x_{1}, x_{2}\right)$ otherwise.

2) If $b>0$ and $c>0$, when $v\left(x_{1}, x_{2}\right)=k$ for some constant $k$, then the eigenvalues of II.5 are real for all $k$, and II.5 is either exponentially stabilizable via static output feedback or is not stabilizable by any choice of $v\left(x_{1}, x_{2}\right)$.

3) If $b>0$ and $c<0$, when $v\left(x_{1}, x_{2}\right)=k$ for some constant $k$, then the eigenvalues of II.5 are not real for all $k$, and II.5 is exponentially stabilizable either by static output feedback or by feedback of the form

$$
v\left(x_{1}, x_{2}\right)= \begin{cases}k_{1}, & \text { if } w_{1}^{\prime} x=0 \\ k_{2}, & \text { if } w_{1}^{\prime} x \neq 0\end{cases}
$$

for some appropriate choice of $w_{1}, k_{1}$ and $k_{2}$.

We prove each part separately below.

Proof of Part 1: Note that if $b=0$, the system described by II.4 has an uncontrollable mode. In this case, stabilizability is possible if and only if $a<0$ and can be achieved via $v\left(x_{1}, x_{2}\right)=k$, where $k<0$. In a similar vein, if $c=0$, II.4 has an unobservable mode. Noting that any initial condition with $x_{2}(0)=0$ satisfies $x_{2}(t)=0$ for all $t$, it is again clear that stabilizability is possible if and only if $a<0$ and can be achieved by setting $v\left(x_{1}, x_{2}\right)$ to a negative real constant.

Proof of Part 2: If we set $v\left(x_{1}, x_{2}\right)=k$ for some constant $k$, the characteristic polynomial of II.5 is given by

$$
s^{2}-(a+k) s+a k-b c .
$$

First note that both roots of II.6 are real for any value of $k$ since the discriminant $(a+k)^{2}-4 a k+4 b c=(a-k)^{2}+$ $4 b c>0$ for all $k$. Now, both eigenvalues of II.5 can be placed in the open left half plane if and only if there exists a value of $k$ such that $a+k<0$ and $a k-b c>0$. When $a<0$, there always exists a value of $k$ which satisfies both of these constraints and, hence, II.5 is stabilizable via static output feedback.

When $a \geq 0$, there is no value $k$ which can satisfy both inequalities simultaneously when $b>0$ and $c>0$. Hence, II.5 cannot be stabilized via static output feedback. To show that II.5 cannot be stabilized for any choice of $v\left(x_{1}, x_{2}\right)$, first recognize that, when $b>0$, and $c>0$, the conic region $x_{1}>0, x_{2}>0$ is invariant under the flow of II.5 for any choice of $v\left(x_{1}, x_{2}\right)$. To show this, assume that the statement is not true, and that there exists a trajectory with $x_{1}(0)>0$, $x_{2}(0)>0$ that leaves the open first quadrant by crossing the axis $x_{1}=0$. At the point of time that the trajectory crosses the $x_{1}$ axis, the corresponding value of $\dot{x}_{1}$ is given by $b x_{2}>0$ which means that $x_{1}(t)$ must be increasing when it crosses the $x_{1}$ axis, an obvious contradiction. Similarly, if there exists some choice of $v\left(x_{1}, x_{2}\right)$ such that a trajectory escapes the open first quadrant by crossing the $x_{2}$ axis, at the time of crossing, $\dot{x}_{2}=c x_{1}>0$.

If $a \geq 0, b>0, c>0$, then II.5 is not stabilizable for any choice of $v\left(x_{1}, x_{2}\right)$ for essentially the same reason as 
was presented in Example I.1. By virtue of the above, if $x_{1}(0)>0, x_{2}(0)>0$, then $\dot{x}_{1}=a x_{1}+b x_{2}>0$, which means that $x_{1}(t)$ is always increasing for any choice of $v\left(x_{1}, x_{2}\right)$.

Proof of Part 3: When $c<0$, the roots of II.6 can be made to lie in the open left half plane when $a<0$. When $a \geq 0$, the roots can also be made to lie in the open left half plane if and only if $a^{2}<-b c$. Hence, II.5 is not static output feedback stabilizable if $a^{2} \geq-b c$, yet, as we now show, there exists a choice of $v\left(x_{1}, x_{2}\right)$ which yields global exponential stability. Closer examination of the characteristic polynomial II.6 with $b>0, c<0$, and $a^{2} \geq-b c$ yields the following two statements:

- The roots of II.6 are complex with nonnegative real part whenever $a-2 \sqrt{-b c}<k<a+2 \sqrt{-b c}$.

- There exists a negative real root of II.6 whenever $k<$ $-a$.

Since the roots of II.6 can be calculated explicitly as

$$
s=\frac{a+k}{2} \pm \frac{\sqrt{(a-k)^{2}+4 b c}}{2},
$$

it is clear that the roots are complex whenever the first bulleted item holds. Moreover, the real part of the roots is nonnegative since, due to the fact that $a^{2} \geq-b c$,

$$
\frac{a+k}{2}>a-\sqrt{-b c} \geq 0 .
$$

Now, when $k<-a$, the discriminant satisfies

$$
(a-k)^{2}+4 b c>4 a^{2}+4 b c \geq 0,
$$

hence, the roots are real. Moreover, because the sum of the roots $(a+k) / 2<0$, one root must be negative.

Informally speaking, to find a choice of $v\left(x_{1}, x_{2}\right)$ which asymptotically stabilizes II.5, we use the following basic design strategy. The above analysis shows that there exists a value of $k_{1}$ which yields a real eigenvalue $\lambda_{1}<0$ and corresponding real eigenvector $q_{1}$. If we set $v\left(x_{1}, x_{2}\right)=k_{1}$ along $q_{1}$, then any initial condition which lies along $q_{1}$ will decay exponentially with rate $\lambda_{1}$. For all other values of $x_{1}$ and $x_{2}$ which do not lie along $q_{1}$, we find a value $k_{2}$ for which the eigenvalues are complex. If we set $v\left(x_{1}, x_{2}\right)=k_{2}$ everywhere else in the state-space, then any initial condition which does not lie along $q_{1}$ will rotate until it eventually "hits" $q_{1}$ and will decay exponentially thereafter. This idea is illustrated graphically in Fig. II.1. Here, the dotted line represents the stable eigenvector $q_{1}$ when $v\left(x_{1}, x_{2}\right)=k_{1}$, the dashed line represents a sample phase portrait with initial condition $x(0)$ when $v\left(x_{1}, x_{2}\right)=k_{2}$ throughout the entire state-space, and the solid curve represents the trajectory with initial condition $x(0)$ when $v\left(x_{1}, x_{2}\right)=k_{1}$ along $q_{1}$ and $v\left(x_{1}, x_{2}\right)=k_{2}$ everywhere else in the statespace.

Before we prove this result formally, we need the following lemma:

Lemma II.1: Consider the linear system $\dot{z}=A z$ where $A \in \mathbf{R}^{2 \times 2}$ has two complex conjugate eigenvalues. Then for any $w \in \mathbf{R}^{2}$ and any $z(0)$, there exists $t_{0} \in \mathbf{R}$ such that $w^{\prime} z\left(t_{0}\right)=0$.

Proof: If $w^{\prime} z(0)=0$, then the statement immediately follows. Otherwise, without loss of generality, assume that $w^{\prime} z(0)>0$. Because the eigenvalues of $A$ are complex, the entries of the corresponding state transition matrix $\exp (A t)$ are linear combinations of the terms $\exp \left(\sigma_{0} t\right) \cos \left(\omega_{0} t\right)$ and $\exp \left(\sigma_{0} t\right) \sin \left(\omega_{0} t\right)$ where $\sigma_{0}, \omega_{0}>0$. Hence,

$$
w^{\prime} z\left(\frac{\pi}{\omega_{0}}\right)=-\exp \left(\frac{\sigma_{0} \pi}{\omega_{0}}\right) w^{\prime} z(0)<0 .
$$

By continuity of $z(t)$, it then follows that there exists some time $t_{0}<\pi / \omega_{0}$ such that $w^{\prime} z\left(t_{0}\right)=0$.

We now formally prove that the above informal description yields an exponentially stable system.

Proposition II.2: For the system II.5 with $b>0, c<0$, and $a^{2} \geq-b c$, suppose that $k_{1}$ is chosen such that II.5 with $v\left(x_{1}, x_{2}\right)=k_{1}$ has a stable eigenvector $q_{1}$ with corresponding eigenvalue $\lambda_{1}<0$, and $k_{2}$ is chosen such that II.5 has two complex eigenvalues. Let $w_{1}$ satisfy $w_{1}^{\prime} q_{1}=0$ and consider

$$
v\left(x_{1}, x_{2}\right)=\left\{\begin{array}{ll}
k_{1}, & \text { if } w_{1}^{\prime} x=0 \\
k_{2}, & \text { if } w_{1}^{\prime} x \neq 0
\end{array} .\right.
$$

Then II.5 is globally exponentially stable for the above choice of $v\left(x_{1}, x_{2}\right)$ with decay rate $\lambda_{1}$.

Proof: If $x(0)=\alpha q_{1}$ for some $\alpha \in \mathbf{R}$, then $x(t)=\exp \left(\lambda_{1} t\right) x(0)$ and the statement holds. Otherwise, $w_{1}^{\prime} x(0) \neq 0$, and, by virtue of Lemma II.1, there exists some value of $t_{0}$ such that $w_{1}^{\prime} x\left(t_{0}\right)=0$. Now, $x(t)=$ $\exp \left(\lambda_{1}\left(t-t_{0}\right)\right) x\left(t_{0}\right)$ for all $t>t_{0}$.

\section{B. Case 2}

Now we assume a system of the following structure:

$$
A=\left[\begin{array}{ll}
a & b \\
0 & c
\end{array}\right], B=\left[\begin{array}{l}
0 \\
1
\end{array}\right], C=\left[\begin{array}{ll}
1 & 0
\end{array}\right]
$$

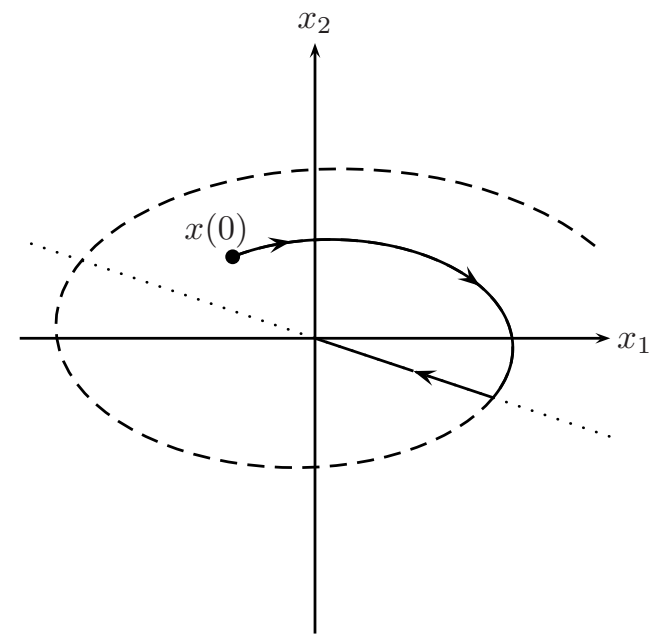

Fig. II.1. Illustration of stabilization algorithm for a system which is not static output feedback stabilizable. 
where $a, c \in \mathbf{R}$, and $b \geq 0$. Here, I. 3 takes the form

$$
\left[\begin{array}{l}
\dot{x}_{1} \\
\dot{x}_{2}
\end{array}\right]=\left[\begin{array}{cc}
a & b \\
v\left(x_{1}, x_{2}\right) & c
\end{array}\right]\left[\begin{array}{l}
x_{1} \\
x_{2}
\end{array}\right]
$$

We summarize the possibilities for stabilizability as a function of the parameters $a, b$, and $c$ in the proposition below:

Proposition II.3: For the system II.9:

1) If $b=0$, then II.9 is exponentially stabilizable via static output feedback if $a<0$ and $c<0$ and is not stabilizable for any choice of $v\left(x_{1}, x_{2}\right)$ otherwise.

2) If $b>0$, when $v\left(x_{1}, x_{2}\right)=k$ for some constant $k$, the eigenvalues of II.9 are not real for all $k$, and II. 9 is exponentially stabilizable either by static output feedback or by feedback of the form

$$
v\left(x_{1}, x_{2}\right)= \begin{cases}k_{1}, & \text { if } w_{1}^{\prime} x=0 \\ k_{2}, & \text { if } w_{1}^{\prime} x \neq 0\end{cases}
$$

for some appropriate choice of $w_{1}, k_{1}$ and $k_{2}$.

We prove each part separately below.

Proof of Part 1: If $b=0$, the system described by II.8 is both uncontrollable and unobservable. In this case, II.9 is stabilizable if and only if $a<0$ and $c<0$. That II.9 is unstable if $a \geq 0$ is clear; if $c \geq 0$, then any solution with initial condition $x_{1}(0)=0$ satisfies $\dot{x}_{2}=c x_{2}$ and, hence, II.9 is unstable for any choice of $v\left(x_{1}, x_{2}\right)$.

Proof of Part 2: If we set $v\left(x_{1}, x_{2}\right)=k$ for some constant $k$, the characteristic polynomial of II.9 is

$$
s^{2}-(a+c) s+a c-b k .
$$

It is clear that if $a+c<0$, then there always exists a choice of $k$ such that $a c-b k>0$, and hence II.9 can be stabilized via static output feedback. If $a+c \geq 0$, then II.9 can be stabilized via a choice of $v\left(x_{1}, x_{2}\right)$ which takes on two values throughout the entire state space in a manner similar to that of Case 1. A more detailed observation of the roots of II.10 when $a+c \geq 0$ reveal the following two facts:

- The roots of II.10 are complex with nonnegative real part whenever $k<-(a-c)^{2} / 4 b$.

- There exists a negative real root of II.10 whenever $k>$ $a c / b$.

Because the roots of II.10 can be calculated explicitly as

$$
s=\frac{a+c}{2} \pm \frac{\sqrt{(a-c)^{2}+4 b k}}{2},
$$

it is clear that the roots are complex whenever the first bulleted item holds.

Now, if $k$ is chosen such that a negative real root exists, then the inequality $a+c<\sqrt{(a-c)^{2}+4 b k}$ must be satisfied. A simple calculation shows that this is equivalent to the second bulleted item.

Using this result, we can derive a stabilization algorithm which is completely analogous to the algorithm of the previous case:
Proposition II.4: For the system II.9 with $b>0$ and $a+$ $c \geq 0$, suppose that $k_{1}$ is chosen such that II.9 has a stable eigenvector $q_{1}$ with corresponding eigenvalue $\lambda_{1}<0$, and $k_{2}$ is chosen such that II.9 has two complex eigenvalues. Let $w_{1}$ satisfy $w_{1}^{\prime} q_{1}=0$, and consider

$$
v\left(x_{1}, x_{2}\right)=\left\{\begin{array}{ll}
k_{1}, & \text { if } w_{1}^{\prime} x=0 \\
k_{2}, & \text { if } w_{1}^{\prime} x \neq 0
\end{array} .\right.
$$

Then II.5 is globally exponentially stable for the above choice of $v\left(x_{1}, x_{2}\right)$ with decay rate $\lambda_{1}$.

Proof: Same as the proof of Proposition II.2.

\section{MAin RESUlT}

While the case studies of the prior section may seem constrained due to the very special structure of the $A, B$, and $C$ matrices, an appropriate change of coordinates reveals that any second order system of the form I.1 and can be transformed into either Case 1 or Case 2.

Lemma III.2: Consider matrices $A \in \mathbf{R}^{2 \times 2}, B \in \mathbf{R}^{2 \times 1}$, and $C \in \mathbf{R}^{1 \times 2}$ where neither $B$ nor $C$ is identically 0 . For any invertible matrix $T \in \mathbf{R}^{2 \times 2}$, define the triplet $(\tilde{A}, \tilde{B}, \tilde{C})$ as $\left(T^{-1} A T, T^{-1} B, C T\right)$, and let

$$
\tilde{A} \equiv\left[\begin{array}{ll}
a & b \\
c & d
\end{array}\right] \text {. }
$$

Then the following statements hold:

1) If $C B \neq 0$, then $\exists T$ such that

$$
\tilde{B}=\left[\begin{array}{l}
0 \\
1
\end{array}\right], \tilde{C}=\left[\begin{array}{ll}
0 & \alpha
\end{array}\right]
$$

with $\alpha \neq 0$ and $b \geq 0$.

2) If $C B=0$, then $\exists T$ such that

$$
\tilde{B}=\left[\begin{array}{l}
0 \\
1
\end{array}\right], \tilde{C}=\left[\begin{array}{ll}
\alpha & 0
\end{array}\right]
$$

with $\alpha \neq 0$ and $b \geq 0$.

Proof: $\quad$ Let $B=\left[\begin{array}{ll}\beta_{1} & \beta_{2}\end{array}\right]^{\prime}, C=\left[\begin{array}{ll}\gamma_{1} & \gamma_{2}\end{array}\right]$. To prove the first result, direct computation shows that the matrix

$$
T=\left[\begin{array}{rr}
\gamma_{2} & \beta_{1} \\
-\gamma_{1} & \beta_{2}
\end{array}\right]
$$

is invertible since $\operatorname{det}(T)=\gamma_{1} \beta_{1}+\gamma_{2} \beta_{2}=C B \neq 0$. Moreover, $\tilde{B}=\left[\begin{array}{ll}0 & 1\end{array}\right]^{\prime}, \tilde{C}=\left[\begin{array}{ll}0 & \alpha\end{array}\right]$ where $\alpha=$ $C B \neq 0$. If $b \geq 0$, then the statement follows. Otherwise, the transformation

$$
T_{2}=T\left[\begin{array}{rr}
-1 & 0 \\
0 & 1
\end{array}\right]=\left[\begin{array}{rr}
-\gamma_{2} & \beta_{1} \\
\gamma_{1} & \beta_{2}
\end{array}\right]
$$

will satisfy all of the desired properties.

To prove the second part of the statement, consider the matrix

$$
T=\left[\begin{array}{rr}
\beta_{2} & \beta_{1} \\
-\beta_{1} & \beta_{2}
\end{array}\right] .
$$

Then $\operatorname{det}(T)=\beta_{1}^{2}+\beta_{2}^{2} \neq 0$, and, hence, $T$ is invertible. Note that any nonzero $C$ which satisfies $C B=0$ may be 
written as $C=\left[\begin{array}{ll}\delta \beta_{2} & -\delta \beta_{1}\end{array}\right]$, where $\delta \neq 0$. Hence, $\tilde{B}=\left[\begin{array}{ll}0 & 1\end{array}\right]^{\prime}, \tilde{C}=\left[\begin{array}{ll}\alpha & 0\end{array}\right]$, where $\alpha=\delta\left(\beta_{1}^{2}+\beta_{2}^{2}\right) \neq$ 0 . If $b \geq 0$, then the statement holds. Otherwise, the transformation

$$
T_{2}=T\left[\begin{array}{rr}
-1 & 0 \\
0 & 1
\end{array}\right]=\left[\begin{array}{rr}
-\beta_{2} & \beta_{1} \\
\beta_{1} & \beta_{2}
\end{array}\right]
$$

will satisfy all of the desired properties.

We are now ready to present the main result of the paper.

Theorem III.1: Consider the system I.1 with $A \in$ $\mathbf{R}^{2 \times 2}, B \in \mathbf{R}^{2 \times 1}$, and $C \in \mathbf{R}^{1 \times 2}$ where neither $C$ nor $B$ is identically 0 . Define the root locus of this system to be the locus of eigenvalues of I.3 when $v\left(x_{1}, x_{2}\right)=k$ as $k$ varies continuously over $\mathbf{R}$. Then exactly one of the following statements is true:

1) The system is static output feedback stabilizable.

2) The system is not static output feedback stabilizable, but it has root locus which takes on complex values for some values of $k \in \mathbf{R}$ and is stabilizable by a control law $v\left(x_{1}, x_{2}\right)$ which takes on one of two values throughout the entire state space.

3) The system has a root locus which is real for all values of $k \in \mathbf{R}$ and is not stabilizable by control of the form I. 3 for any choice of $v\left(x_{1}, x_{2}\right)$.

Proof: Using Lemma III.2, whenever $C$ and $B$ are not identically 0 , there exists a coordinate transformation where I. 3 is either of the form

$$
\left[\begin{array}{c}
\dot{x}_{1} \\
\dot{x}_{2}
\end{array}\right]=\left[\begin{array}{cc}
a & b \\
c & d+\alpha v\left(x_{1}, x_{2}\right)
\end{array}\right]\left[\begin{array}{l}
x_{1} \\
x_{2}
\end{array}\right]
$$

or the form

$$
\left[\begin{array}{l}
\dot{x}_{1} \\
\dot{x}_{2}
\end{array}\right]=\left[\begin{array}{cc}
a & b \\
c+\alpha v\left(x_{1}, x_{2}\right) & d
\end{array}\right]\left[\begin{array}{l}
x_{1} \\
x_{2}
\end{array}\right],
$$

with $\alpha \neq 0$ and $b \geq 0$. Since $\alpha \neq 0$, the substitutions $\tilde{u}\left(x_{1}, x_{2}\right)=d+\alpha v\left(x_{1}, x_{2}\right)$ and $\tilde{u}\left(x_{1}, x_{2}\right)=c+\alpha v\left(x_{1}, x_{2}\right)$ are invertible. Hence, any system of the form I.1 for which neither $C$ nor $B$ is identically 0 can be transformed into the form of either Case 1 or Case 2 of the previous section. Since the statements of the theorem were shown to be true for both of these case studies, it then follows that the result must hold in the more general setting.

\section{Design Methodology}

Note that in order to obtain a stabilizing controller (when it exists), one need not carry out the transformations described in Lemma III.2. Rather, one may analyze the root locus of the matrix $A+k B C$ directly and (when necessary) find a stable eigenvector to derive an appropriate control law $v\left(x_{1}, x_{2}\right)$. Moreover, when $(A, B)$ is reachable and $(C, A)$ is observable, we may employ classical root locus techniques to the corresponding transfer function $C(s I-A)^{-1} B$ to quickly ascertain the geometric behavior of the root locus. When either $(A, B)$ is not reachable and/or $(C, A)$ is not observable, we may still use classical root locus techniques on the transfer function $C(s I-A)^{-1} B$, but we must take care to include the unreachable and/or unobservable modes in our analysis.

The following basic algorithm will yield a stabilizing controller when one exists:

1) Compute the transfer function $C(s I-A)^{-1} B$ and examine the corresponding root locus of I.1 (i.e. the roots of $1-k C(s I-A)^{-1} B$ as $k$ varies over $\mathbf{R}$, along with any fixed unreachable and/or unobservable modes of the original state-space model).

2) If examination of the root locus shows that $\exists k_{0}$ for which both of the eigenvalues of $A+k_{0} B C$ lie in the open left half-plane, find such a value of $k_{0}$ and choose $v\left(x_{1}, x_{2}\right)=k_{0}$ for all $x$.

3 ) If examination of the root locus indicates that there exists a value $k_{1}$ for which one of the eigenvalues $A+k_{1} B C$ lies in the open left half-plane and a value $k_{2}$ for which the imaginary part of the eigenvalues is nonzero, find corresponding values of $k_{1}$ and $k_{2}$, along with the (real) eigenvector $w_{1}$ of $A+k_{1} B C$ corresponding to the stable eigenvalue. Choose $v\left(x_{1}, x_{2}\right)$ such that

$$
v\left(x_{1}, x_{2}\right)= \begin{cases}k_{1}, & \text { if } w_{1}^{\prime} x=0 \\ k_{2}, & \text { if } w_{1}^{\prime} x \neq 0\end{cases}
$$

where $w_{1}$ satisfies $w_{1}^{\prime} q_{1}=0$.

4) If neither 2) nor 3) holds, declare the system unstabilizable by any choice of $v\left(x_{1}, x_{2}\right)$.

\section{EXAMPLES}

Example V.2: We consider three reachable, observable systems of the form

$$
\begin{gathered}
\dot{x}=A_{i} x+B_{i} u, \quad y=C_{i} x, \quad i \in\{1,2,3\} \\
A_{1}=\left[\begin{array}{rr}
-6 & -6 \\
-6 & 7
\end{array}\right] \quad A_{2}=\left[\begin{array}{ll}
0 & 1 \\
6 & 1
\end{array}\right] \quad A_{3}=\left[\begin{array}{rr}
0 & 1 \\
-12 & 7
\end{array}\right] \\
B_{1}=\left[\begin{array}{r}
-1 \\
1
\end{array}\right] \quad B_{2}=\left[\begin{array}{l}
0 \\
1
\end{array}\right] \quad B_{3}=\left[\begin{array}{l}
0 \\
1
\end{array}\right] \\
C_{1}=\left[\begin{array}{ll}
0 & 1
\end{array}\right] \quad C_{2}=\left[\begin{array}{ll}
1 & 1
\end{array}\right] \quad C_{3}=\left[\begin{array}{ll}
-2 & 1
\end{array}\right] .
\end{gathered}
$$

The transfer functions $H_{i}(s)$ corresponding to each of these state space descriptions are given by

$$
\begin{aligned}
& H_{1}(s)=\frac{s}{s^{2}-s-6} \\
& H_{2}(s)=\frac{s+1}{s^{2}-s-6} \\
& H_{3}(s)=\frac{s-2}{s^{2}-7 s+12}
\end{aligned}
$$

The root locus for each of the above transfer functions is depicted in Fig. V.2. From the first root locus diagram for $H_{1}(s)$, it is clear that the root locus is real for all $k$, but the zero at $s=0$ prevents one eigenvalue from entering the left half plane. Hence, there is no switching control law of the form I.2 which can asymptotically stabilize this system. 
While the root locus for $H_{2}(s)$ is also real for all $k$, the presence of the zero at $s=-1$ allows both eigenvalues to lie in the open left half plane for sufficiently negative values of $k$. Indeed, when $k=-7$, the eigenvalues are approximately -5.83 and -0.17 . Hence, the second system can be made stable via static output feedback.

The third system $H_{3}(s)$ has a root locus that takes on complex values for some negative values of $k$, but both eigenvalues never lie in the left half plane simultaneously. Nevertheless, one of the eigenvalues can be made negative for sufficiently negative values of $k$. Indeed, when $k=$ $-20 / 3,-1$ is an eigenvalue of $A_{3}+k B_{3} C_{3}$ with corresponding eigenvector $q_{1}=\left[\begin{array}{ll}1 & -1\end{array}\right]^{\prime}$. When $k=-1$, the eigenvalues of $A_{3}+k B_{3} C_{3}$ are complex $(3 \pm i)$. Noting that $w_{1}=\left[\begin{array}{ll}1 & 1\end{array}\right]^{\prime}$ satisfies $w_{1}^{\prime} q_{1}=0$, a stabilizing switching controller is given by $u\left(x_{1}, x_{2}\right)=v\left(x_{1}, x_{2}\right) C_{3} x$, where $v\left(x_{1}, x_{2}\right)$ is given by

$$
v\left(x_{1}, x_{2}\right)=\left\{\begin{array}{ll}
-\frac{20}{3}, & \text { if } x_{1}+x_{2}=0 \\
-1, & \text { if } x_{1}+x_{2} \neq 0
\end{array} .\right.
$$

The purpose of the root locus diagrams in the previous example is to illustrate how one can almost immediately tell whether stability can be achieved by a single gain, two gains, or by no control of the form $u\left(x_{1}, x_{2}\right)=$ $v\left(x_{1}, x_{2}\right) C x$. Using the standard root locus techniques on the transfer function $C(s I-A)^{-1} B$, it is fairly quick and easy to determine the basic geometric features of the locus and, hence, determine in which of the three stabilization categories a given system lies.

Example V.3: We now consider two unreachable systems
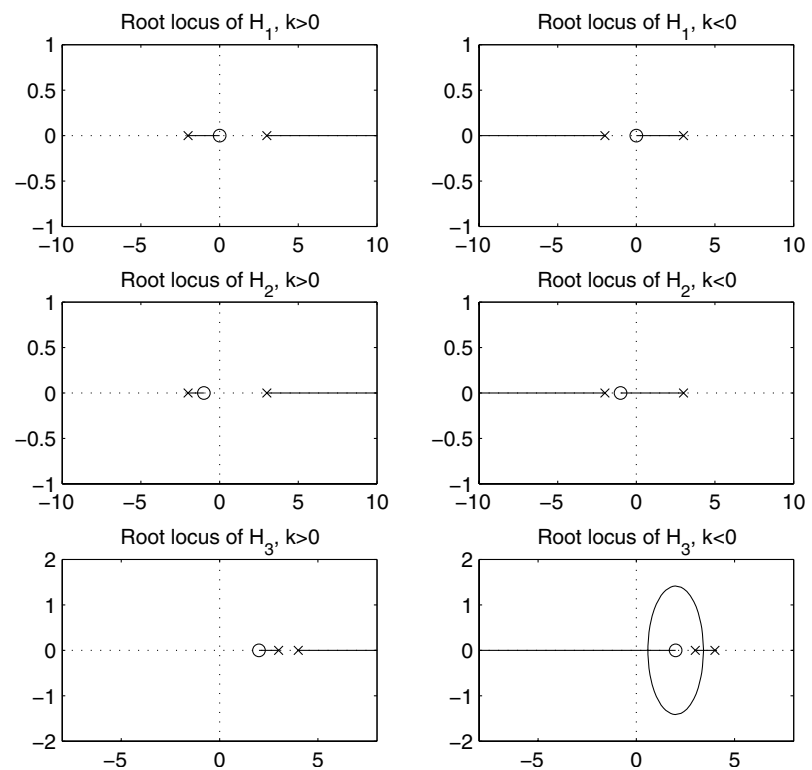

Fig. V.2. Root loci for $H_{1}(s), H_{2}(s)$, and $H_{3}(s)$. The root loci for positive $k$ are depicted on the left, while the root loci for negative $k$ are depicted on the right. of the form

$$
\dot{x}=A_{i} x+B u, \quad y=C x,
$$

where $B=\left[\begin{array}{ll}1 & 0\end{array}\right], C=\left[\begin{array}{ll}1 & 1\end{array}\right]$ and

$$
A_{1}=\left[\begin{array}{rr}
-1 & 1 \\
0 & 1
\end{array}\right], A_{2}=\left[\begin{array}{rr}
-1 & 1 \\
0 & -1
\end{array}\right] \text {. }
$$

In both cases, the transfer function $C\left(s I-A_{i}\right)^{-1} B=$ $1 /(s+1)$, from which it is clear that the root locus lies along the negative real line for an appropriately chosen value of the gain $k$. However, since the root locus of the entire system is given by the root locus of the transfer function united with the fixed, unreachable modes, only the second system is stabilizable in this case since the unreachable mode lies in the open left half plane. The first system has a root locus which is real for all $k$, but an unstable eigenvalue at $s=1$ always exists. Hence, no feedback of the form $u\left(x_{1}, x_{2}\right)=v\left(x_{1}, x_{2}\right) C x$ can stabilize the first system for any $v\left(x_{1}, x_{2}\right)$.

\section{CONCLUSion ANd Future Work}

Some remarks are in order. First, the switching law presented in this paper is not implementable from a practical standpoint since the value of the gain is constant everywhere except on a measure zero set. Nevertheless, with the addition of a finite state automaton and appropriate modifications to the switching law, a robust version of this switching law can be implemented and is the subject of future work.

Second, while it is arguable that a connection between stabilizability with the given controller structure and the geometric behavior of the corresponding root locus is strong only because of the simplistic nature of second order systems, preliminary work indicates that a connection still exists between the two for higher dimensional systems. Indeed, for a certain class of higher dimensional systems, there is an indication that the root locus provides certain negative results in the sense that a system cannot be stabilized by control of the form $u(x)=v(x) C x$ for any $v(x)$ if the root locus possesses certain geometric properties similar to the geometric properties of the second order case presented here.

\section{REFERENCES}

[1] Z. Artstein, Example of Stabilization with Hybrid Feedback, in Hybrid Systems III: Verification and Control, Lecture Notes in Computer Science 1066, Springer-Verlag, Berlin, 1996, pp. 173-185.

[2] E. Litsyn, Y. V. Nepomnyashchikh and A. Posonov, Stabilization of Linear Differential Systems via Hybrid Feedback Controls, SIAM Journal of Control Optimization Vol. 38, No. 5, pp. 1468-1480, 2000.

[3] B. Hu, G. Zhai and A. N. Michel, Hybrid Output Feedback Stabilization of Two-Dimensional Linear Control Systems, Proceedings of the 2000 American Control Conference, pp. 2184-2188, Chicago, Illinois, June, 2000.

[4] X. Xu and P. J. Antsaklis, Design of Stabilizing Control Laws for Second-order Switched Systems, Proceedings of the 14th IFAC World Congress, vol. C., pp. 181-186, Beijing, P.R. China, July 1999.

[5] X. Xu and P. J. Antsaklis, Stabilization of Second-order LTI Switched Systems, ISIS Technical Report isis-99-001, Dept. of Elec. Enging., University of Notre Dame, January, 1999. 